\title{
SPIN COBORDISM
}

BY D. W. ANDERSON, E. H. BROWN, JR., AND F. P. PETERSON ${ }^{1}$

Communicated by I. Singer, October 20, 1965

1. Statements of results. $\Omega_{*}^{\text {Spin }}$, the Spin cobordism ring, has been studied by many, e.g. Wall [9, p. 294], Milnor [5] and [6], Novikov [7], and P. G. Anderson [3]. In this announcement we describe the additive structure of $\Omega_{*}^{\text {Spin }}$, much of the multiplicative structure, characteristic numbers which determine $\Omega_{*}^{\text {Spin }}$, and other properties.

We first state some technical results. Let $Q$ denote the mod 2 Steenrod algebra, and let $Q_{0}=S q^{1}$ and $Q_{1}=S q^{3}+S q^{2} S q^{1}$. If $a_{1}, a_{2}, \cdots, a_{r}$ $\in \mathfrak{Q}, \mathfrak{Q}\left(a_{1}, a_{2}, \cdots, a_{r}\right)$ will denote the left ideal generated by $\left\{a_{i}\right\}$. All cohomology groups will have $Z_{2}$ coefficients unless otherwise stated. Let $p: B O\langle n\rangle \rightarrow B O$ be the fibre space such that $\pi_{i}(B O\langle n\rangle)=0$ for $i<n$ and $p_{*}: \pi_{i}(B O\langle n\rangle) \approx \pi_{i}(B O)$ for $i \geqq n$. The following theorem is due to R. Stong [8].

THEOREM 1.1. There is an element $\alpha_{n} \in H^{n}(B O\langle n\rangle)$ such that the map of $a$ into $\bar{H}^{*}(B O\langle n\rangle)$ given by $a \rightarrow a \alpha_{n}$ defines an isomorphism in dimensions less than $2 n$ between $Q / Q\left(S q^{1}, S q^{2}\right)$ and $\bar{H}^{*}(B O\langle n\rangle)$ for $n \equiv 0(\bmod$ 8) and between $Q / Q\left(S q^{8}\right)$ and $\bar{H}^{*}(B O\langle n\rangle)$ for $n \equiv 2(\bmod 8)$.

Let $\xi \in \bar{K} O^{\circ}(X)(X)$ be of filtration $n$ [4], that is, $\xi$ is trivial on the $n-1$ skeleton of $X$. Then there is a map $f_{\xi}: X \rightarrow B O\langle n\rangle$ such that $p f_{\xi}$ is $\xi$. Let $[\xi]=\left\{f_{\xi}^{*}\left(\alpha_{n}\right)\right\} \subset H^{n}(X)$ for all $f_{\xi}$ such that $p f_{\xi}=\xi$.

Let $J=\left(j_{1}, \cdots, j_{k}\right)$ be a sequence of integers with $j_{i}>1$ and $k \geqq 0$. Let $P_{J}=P_{j_{1}} \cdots P_{j_{k}} \in H^{4 n(J)}\left(B\right.$ Spin), where $n(J)=\sum j_{i}$ and $P_{j}$ is the $j$ th Pontrjagin class. In [2], certain classes $\pi^{i} \in K O^{\circ}(B S O)$ were defined which behave very much like Pontrjagin classes. Under the map $B$ Spin $\rightarrow B S O, \pi^{i}$ maps into a class which we also denote $\pi^{i} \in K O^{0}(B$ Spin $)$. Let $\pi^{J}=\pi^{j_{1}} \cdots \pi^{j_{k}} \in K O^{0}(B$ Spin $)$. Our main result from $K O$-theory is the following theorem.

THEOREM 1.2. The filtration of $\pi^{J}$ is $4 n(J)$ if $n(J)$ is even, and is $4 n(J)-2$ if $n(J)$ is odd. Furthermore, if $n(J)$ is even, there exists $X_{J} \in H^{4 n(J)}\left(B\right.$ Spin) such that $X_{J} \in\left[\pi^{J}\right]$ and $X_{J} \equiv P_{J} \bmod \operatorname{Im} Q_{0} Q_{1}$, and if $n(J)$ is odd, there exists $Y_{J} \in H^{4 n(J)-2}\left(B\right.$ Spin) such that $Y_{J} \in\left[\pi^{J}\right]$ and $S q^{2} Y_{J} \equiv P_{J} \bmod \operatorname{Im} Q_{0} Q_{1}$.

${ }^{1}$ The first named author was partially supported by the U.S.A.F. as a N.A.S.N.R.C. fellow and by the U. S. Army Research Office, the second named author by the N.S.F. and the third named author by the N.S.F. and the U. S. Army Research Office. 
Let $M \operatorname{Spin}(n)$ denote the universal Thom complex for $\operatorname{Spin}(n)$ bundles, and let $M$ Spin denote the spectrum whose $n$th term is $M \operatorname{Spin}(n)$. We define $\bar{H}^{i}(M \operatorname{Spin})$ to be lim inv $\bar{H}^{n+i}(M \operatorname{Spin}(n))$. We let $X_{J}, Y_{J}$, and $P_{J} \in \bar{H}^{*}(M$ Spin $)$ denote the elements corresponding under the Thom isomorphism to $X_{J}, Y_{J}$, and $P_{J} \in H^{*}(B$ Spin). Likewise, we let $\pi^{J} \in \mathrm{Cl}(K O)^{\circ}(M \mathrm{Spin})$ correspond under the $K O$ Thom isomorphism to $\pi^{J} \in K O^{\circ}(B$ Spin $)$.

Let $X$ and $Y$ be the graded vector spaces over $Z_{2}$ generated, respectively, by $\left\{X_{J}\right\}, n(J)$ even, and $\left\{Y_{J}\right\}, n(J)$ odd. The following theorem gives the structure of $H^{*}(M \operatorname{Spin})$ as a module over $a$.

Theorem 1.3. There is a graded vector space $Z$ and an a-module isomorphism

$\theta:\left(Q / Q\left(S q^{1}, S q^{2}\right) \otimes X\right) \oplus\left(Q / Q\left(S q^{3}\right) \otimes Y\right) \oplus(Q \otimes Z) \rightarrow H^{*}(M$ Spin $)$

such that $\theta\left(X_{J}\right)=X_{J}$ and $\theta\left(Y_{J}\right)=Y_{J}$.

Let $B O\langle n\rangle$ denote the $\Omega$-spectrum whose 0 th term is $B O\langle n\rangle$. If $\boldsymbol{n}(J)$ is even let $f_{J}: M$ Spin $\rightarrow B O\langle 4 n(J)\rangle$ be a map corresponding to $\pi^{J}$ such that $f_{J}^{*}\left(\alpha_{4 n(J)}\right)=X_{J}$ and, if $n(J)$ is odd, let $f_{J}: M$ Spin $\rightarrow B O\langle 4 n(J)-2\rangle$ be a map corresponding to $\pi^{J}$ such that $f_{J}^{*}\left(\alpha_{4 n(j)-2}\right)$ $=Y_{J}$. Let $\left\{Z_{i}\right\}$ be a basis for $Z$ and let $f_{i}: M \operatorname{Spin} \rightarrow \boldsymbol{K}\left(Z_{2}, \operatorname{dim} Z_{i}\right)$ be a map corresponding to $Z_{i}\left(K\left(Z_{2}, n\right)\right.$ is the spectrum whose 0 th term is $K\left(Z_{2}, n\right)$ ). Let

$$
\begin{aligned}
F: M \operatorname{Spin} & \rightarrow \prod_{n(J) \text { even }} B O\langle 4 n(J)\rangle \times \prod_{n(J) \text { odd }} B O\langle 4 n(J)-2\rangle \\
& \times \prod^{K\left(Z_{2}, \operatorname{dim} Z_{i}\right)}
\end{aligned}
$$

be given by $F=\prod f_{J} \times \prod f_{i}$.

CoROLlaRy 1.4. F induces an isomorphism on cohomology mod 2 and hence an isomorphism mod $\mathfrak{C}_{2}$ on homotopy groups, where $\mathfrak{C}_{2}$ is the class of finite groups of odd order.

We now give some of the geometric corollaries of the above theorems. If $[M] \in \Omega_{n}^{\text {Spin }}, \pi^{J}(M) \in K O^{-n}(p t)$ denotes the characteristic number defined by $\pi^{J}$ (see [2]).

CoRollary 1.5. Let $[M] \in \Omega_{*}^{\text {Spin }}$. Then $[M]=0$ if and only if $\pi^{J}(M)$ $=0$ for all $J$ and all Stiefel-Whitney numbers of $M$ vanish.

Corollary 1.6. Let $[M] \in \mathfrak{N}_{*}$. Then $[M]$ contains a Spin manifold if and only if all Stiefel-Whitney numbers of $M$ involving $W_{1}$ and $W_{2}$ vanish. 
Theorem 1.7. Let $\Re_{*}=\operatorname{Im}\left(\Omega_{*}^{\text {Spin }} \rightarrow \mathfrak{N}_{*}\right) / \operatorname{Im}$ (squares in $\Omega_{*} \rightarrow \Re_{*}$ ). Then $\Re_{n}=0, n \leqq 28, n \neq 24 . \Re_{24}=Z_{2}=\Re_{29}, \Re_{30}=\Re_{31}=0, \Re_{32}=Z_{2}+Z_{2}$, $\Re_{33}=Z_{2}, \Re_{34}=Z_{2}, \Re_{35}=0, \Re_{36}=Z_{2}, \Re_{37}=Z_{2}+Z_{2}, \Re_{38}=0, \Re_{39}=Z_{2}+Z_{2}$, $\Re_{40}=Z_{2}+Z_{2}+Z_{2}+Z_{2}+Z_{2}, \quad \Re_{41}=Z_{2}+Z_{2}+Z_{2}, \Re_{42}=Z_{2}+Z_{2}+Z_{2}, \quad \Re_{43}$ $=Z_{2}+Z_{2}, \Re_{44}=Z_{2}+Z_{2}+Z_{2}$. (Compare $[5]$ and $[3]$.)

The authors would like to thank Charles Sims who programmed the hard part of the above computation on an IBM 7094. These were done before we proved our main theorems and were helpful in checking our conjectures; in particular they led to the correct version of Theorem 1.3, and they gave us explicit Stiefel-Whitney numbers which detect elements in $\Re_{n}, n \leqq 44$.

Let $n(J)$ be even. Then there are elements $\left[M_{J}\right] \in \Omega_{4 n(J)}^{\mathrm{Spin}}$ of infinite order which are detected by $\left\{P_{J}\right\} \bmod 2$. If $J=\left(j_{1}, \cdots, j_{k}\right)$ has only even integers, we may take $M_{J}=Q P^{i_{1}} \times \cdots \times Q P^{j_{k}}$ where $Q P^{n}$ is the quaterionic projective $n$ space. Let $n(J)$ be odd. Then there are elements $\left[N_{J}\right] \in \Omega_{4 n(J)-2}^{\text {Spin }}$ of order two which are detected by $\left\{Y_{J}\right\}$. Note $\left[N_{J}\right] \times \alpha=0$, where $0 \neq\left[\bar{S}^{1}\right]=\alpha \in \Omega_{1}^{\mathrm{Spin}}=Z_{2}$ by Corollary 1.5. Let $\partial W_{J}=N_{J} \times \bar{S}^{1}$, and let $\partial V_{2}=\bar{S}^{1} \times S^{0}$. Then $\partial\left(W_{J} \times S^{0}\right)=\partial\left(N_{J} \times V_{2}\right)$. Let $M_{J}=W_{J} \times S^{0} \cup N_{J} \times V_{2}$. Then $\left[M_{J}\right] \in \Omega_{4 n(J)}^{\text {Spin }}$ (the indeterminacy in this construction is all multiples of two). Let $\tau \in \Omega_{4}^{\text {Spin }}=Z$ be a generator, and let $\omega \in \Omega_{8}^{\text {Spin }}=Z \oplus Z$ be an element such that $\hat{A}(\omega)=1$ (see [6]).

Corollary 1.8. A basis for $\Omega_{*}^{\text {Spin }} \otimes Q$ is given by $\left[M_{J}\right] \times \omega^{k}$ and $\left[M_{J}\right] \times \tau \times \omega^{k}, k=0,1, \cdots$.

CoRollary 1.9. $\operatorname{Ker}\left(\Omega_{*}^{\text {Spin }} \rightarrow \Omega_{*}\right)$ is a vector space over $Z_{2}$ with a basis given by $\left[M_{J}\right] \times \omega^{k} \times \alpha^{i}, k=0,1, \cdots, i=1,2, n(J)$ even and $\left(\left[M_{J}\right] \times \tau\right) / 4 \times \omega^{k} \times \alpha^{i}, k=0,1, \cdots, i=1,2, n(J)$ odd.

Let $\left[M_{i}\right] \in \Omega_{\operatorname{dim} z_{i}}^{\mathrm{Spin}}$ correspond to $Z_{i}$.

Corollary 1.10. $A$ basis for $\Omega_{*}^{\text {Spin }} \otimes Z_{2}$ is given by

(1) $\left[M_{J}\right] \times \omega^{k} \times \alpha^{i}, k=0,1, \cdots, i=0,1,2, n(J)$ even,

(2) $\left[M_{J}\right] \times \tau \times \omega^{k}, k=0,1, \cdots, n(J)$ even,

(3) $\left[M_{i}\right]$,

(4) $\left[N_{J}\right], n(J)$ odd,

(5) $\left[M_{J}\right] \times \omega^{k}, k=0,1, \cdots, n(J)$ odd, and

(6) $\left(\left(\left[M_{J}\right] \times \tau\right) / 4\right) \times \omega^{k} \times \alpha^{i}, k=0,1, \cdots, i=0,1,2, n(J)$ odd.

The rank of $Z$ in dimension $n$ can be computed inductively from Theorem 1.3 and the information given in the following theorem.

Theorem 1.11. (a) The Poincaré polynomial for $H^{*}(M S p i n)$ is 


$$
\prod_{n>3 ; n \neq 2^{r}+1}\left(1-t^{n}\right)^{-1}
$$

(b) The Poincaré polynomial for $Q$ is

$$
\prod_{n=2^{r}-1 ; r \geq 1}\left(1-t^{n}\right)^{-1} \text {. }
$$

(c) The Poincaré polynomial for $a / \propto\left(S q^{1}, S q^{2}\right)$ is

$$
\prod_{n=2^{r}-1 ; r \geq 3}\left(1-t^{n}\right)^{-1}\left(1-t^{4}\right)^{-1}\left(1-t^{6}\right)^{-1} .
$$

(d) The Poincaré polynomial for $Q / \propto\left(S q^{3}\right)$ is

$$
\prod_{n=2^{r}-1 ; r \geq 3}\left(1-t^{n}\right)^{-1}\left(1-t^{4}\right)^{-1}\left(1-t^{6}\right)^{-1}\left(1+t+t^{2}+t^{3}+t^{4}\right) .
$$

We remark that $\Omega_{*}^{\text {Spin }}$ has no odd torsion (see [7]) and so the above theorems determine $\Omega_{*}^{\text {Spin }}$ as an additive group.

Finally, we comment that we can prove similar theorems for the complex spinor group, Spin ${ }^{C}=$ Spin $\times_{z_{2}} U(1)$. In this case a manifold is cobordant to zero if and only if all its Stiefel-Whitney and Pontrjagin numbers are zero.

2. Techniques of proofs. Let $Q_{1}$ be the subalgebra of $a$ generated by $S q^{0}, S q^{1}$, and $S q^{2}$. Let $\Theta$ be a graded $\alpha_{1}$-module. ${ }^{2}$ Note that $Q_{0}, Q_{1} \in Q_{1}$ and that $Q_{0} Q_{0}=0$ and $Q_{1} Q_{1}=0$. We say that $B$ has isomorphic homologies if $\left(\operatorname{Ker} Q_{0} \cap \operatorname{Ker} Q_{1}\right) /\left(\operatorname{Im} Q_{0} \cap \operatorname{Im} Q_{1}\right) \rightarrow H\left(B, Q_{i}\right)$ is an isomorphism for $i=0,1$. The following theorem is a generalization of a theorem due to Wall [9] and seems to be of interest in itself.

THEOREM 2.1. Let $B$ be an $Q_{1}$-module with isomorphic homologies. Then, as an $Q_{1}$-module, $B$ is isomorphic to a direct sum of four types of $\mathfrak{a}_{1}$-modules, namely $\mathfrak{a}_{1} / \mathfrak{Q}_{1}\left(S q^{1}, S q^{2}\right)=Z_{2}, \mathfrak{Q}_{1} / \mathfrak{Q}_{1}\left(Q_{0}, Q_{1}\right), \mathfrak{a}_{1} / \mathfrak{a}_{1}\left(S q^{3}\right)$, and $a_{1}$.

In order to prove Theorem 1.2 we note that $B=H^{*}(B S O)$ satisfies Theorem 2.1, we use the knowledge of $K O^{*}(B S O)$ [1], and compute in the so-called Atiyah-Hirzebruch spectral sequence for $K O^{*}(B S O)$.

Theorem 1.3 follows from the following algebraic theorem.

TheOREM 2.2. Let $X$ and $Y$ be graded vector spaces over $Z_{2}$. Let $₫$ be a connected coalgebra over $Q$ such that $\operatorname{Ker}(\phi: Q \rightarrow ß)=Q\left(S q^{1}, S q^{2}\right)$, where $\phi(a)=a(1)$. Given $\theta^{\prime}:\left(Q / Q\left(S q^{1}, S q^{2}\right) \otimes X\right) \oplus\left(Q / Q\left(S q^{3}\right) \otimes Y\right) \rightarrow B$ such that $\theta_{*}^{\prime}: H\left(\left(\propto / \propto\left(S q^{1}, S q^{2}\right) \otimes X\right) \oplus\left(\propto / \propto\left(S q^{3}\right) \otimes Y\right), Q_{i}\right) \rightarrow H\left(\otimes, Q_{i}\right)$ is

\footnotetext{
2 We assume that $B_{i}=0$ if $i<0$ and that $B_{i}$ is finitely generated.
} 
an isomorphism for $i=0,1$. Define $Z=B / \operatorname{Im} \theta^{\prime}+\bar{Q} \beta$ and extend $\theta^{\prime}$ to $\theta:\left(a / \propto\left(S q^{1}, S q^{2}\right) \otimes X\right) \oplus\left(a / \propto\left(S q^{3}\right) \otimes Y\right) \oplus(\alpha \otimes Z) \rightarrow B$ in the obvious way. Then $\theta$ is an isomorphism.

The proofs of the corollaries to Theorem 1.3 are not difficult if one applies the techniques developed in [2].

\section{BIBLIOGRAPHY}

1. D. W. Anderson, The real K-theory of classifying spaces, Proc. Nat. Acad. Sci. U.S.A. 51 (1964), 634-636.

2. D. W. Anderson, E. H. Brown, Jr. and F. P. Peterson, SU-cobordism, KOcharacteristics classes, and the Kervaire invariant, Ann. of Math. (to appear).

3. P. G. Anderson, Cobordism classes of squares of orientable manifolds, Bull. Amer. Math. Soc. 70 (1964), 818-819.

4. M. F. Atiyah and F. Hirzebruch, Vector bundles and homogeneous spaces, pp. 7-38, Proc. Sympos. Pure Math. Vol. 3, Amer. Math. Soc., Providence, R. I., 1961.

5. J. W. Milnor, On the Stiefel-Whitney numbers of complex manifolds and of spin manifolds, Topology 3 (1965) 223-230.

6. - Remarks concerning spin manifolds, Differential and Combinatorial Topology, Princeton Univ. Press, Princeton, N. J., 1965; 55-62.

7. S. P. Novikov, Homotopy properties of Thom complexes, Mat. Sb. (N.S.) (99) 57 (1962), 407-442.

8. R. Stong, Determination of $H^{*}\left(B O(k, \cdots, \infty), Z_{2}\right)$, and $H^{*}\left(B U(k, \cdots, \infty), Z_{2}\right)$, Trans. Amer. Math. Soc. 107 (1963), 526-544.

9. C. T. C. Wall, Determination of the cobordism ring, Ann. of Math. 72 (1960), 292-311.

10. - A characterization of simple modules over the Steenrod algebra mod 2, Topology 1 (1962), 249-254.

OXFORD UNIVERSITY,

BRANDEIS UNIVERSITY AND

Massachusetts Institute of Technology 